\title{
ROTATING NEUTRON STARS, PULSARS, AND COSMIC X-RAY SOURCES
}

\author{
WALLACE H. TUCKER \\ Rice University, Houston, Texas, U.S.A.
}

The purpose of this paper is to discuss the relationship between rotating neutron stars, pulsars, and cosmic X-ray sources. The latter may be divided into at least two classes: the sources with large angular diameters, such as the Crab Nebula, and those with small angular diameter, such as Sco X-1. I submit that a basic model, consisting of a rotating neutron star losing mass in the presence of a large magnetic field, can account for both types of X-ray source. The extended sources represent the case where the energy in the 'neutron-star wind' is greater than the magnetic energy. The streaming protons and electrons deposit their energy far out into the nebula in a shock transition region. The relativistic electrons responsible for the extended sources of radio, optical and X-ray emission are produced in the transfer of energy between the protons and electrons in the shock wave, and by magnetic pumping in hydromagnetic waves which are generated by fluctuations in the mass loss rate. The compact sources, such as Sco X-1, represent the other extreme where the magnetic energy dominates, so that no mass loss occurs. The particles are then accelerated and radiate in radiation belts around the neutron star, resulting in a source with a small angular diameter.

Consider first the X-ray source in the Crab Nebula: I am referring only to the extended, steady source; the pulsed emission, amounting to $7 \%$ of the total [1], will not be discussed in what follows.

Of fundamental importance for the theory of the Crab Nebula is the observation that the period of the pulsar located there is increasing at a rate of one part in 2400/ year [2]. This slowing down has been interpreted as being due to a torque on a rotating neutron star [3-6] and implies a rotational energy-loss rate comparable to the luminosity of the entire nebula [7]. This agreement has led to the general view that the two phenomena, the rotational energy-loss rate of the neutron star, and the luminosity of the Crab Nebula, are related. For this to be so, energy must be transferred from the rotating neutron star to relativistic particles and then from relativistic particles to radiation.

The second part of this chain is fairly well understood; relativistic electrons in a magnetic field can radiate very efficiently by means of the synchrotron mechanism, and the observations $[8,9]$ are consistent with this interpretation.

If we accept the synchrotron mechanism, then the observed size of the $\mathrm{X}$-ray source places severe restrictions on the way in which the relativistic electrons are produced, as stressed recently by Burbidge and Hoyle [10]. The observations of Oda et al. [11] show that the projected diameter of the source of low energy X-rays is about 2 light years, for an assumed distance of 5000 light years.

The maximum energy that an electron can have after traversing a distance from 
$r_{0}$ to $r$ in a magnetic field varying as

$$
B_{\perp}=B_{\perp_{0}}\left(r_{0} / r\right)^{n}
$$

is given approximately by

$$
\gamma_{\max }=E_{\max } / m c^{2}=10^{19}(2 n-1) / B_{\perp_{0}} r_{0}\left[1-\left(r_{0} / r\right)^{2 n-1}\right],
$$

where $B_{\perp}=$ component of magnetic field perpendicular to the electron velocity. For the case of a uniform field, $r_{0}=0$, and

$$
\gamma_{\max }=10^{19} / B_{\perp_{0}}^{2} r
$$

For $n \geqslant 1$ and $r \gg r_{0}$.

$$
\gamma_{\max }=2 n \times 10^{19} / B_{\perp_{0}}^{2} r_{0}
$$

which shows that the electron loses most of its energy in the region where the magnetic field is large. On the other hand, in order to generate X-rays with energies of $4 \mathrm{keV}$, electron energies corresponding to

$$
\gamma=10^{6} / 2 B_{\perp}^{1 / 2}
$$

are required. Since the magnetic field is practically uniform and equal to about $5 \times 10^{-4}$ gauss over the region occupied by the X-ray source, energies corresponding to $\gamma=2 \times 10^{7}$ are needed. In addition, Equation (3) shows that the maximum radius for the soft X-ray source is $r_{\max } \cong 2 \times 10^{18} \mathrm{~cm}$, just barely compatible with the observations. Of course, this result is sensitive to the assumed magnetic field; for $B_{\perp}=2 \times 10^{-4}$ gauss, $r_{\max } \approx 7 \times 10^{18} \mathrm{~cm}$. If the electrons are produced in a high field region and injected into the nebula, Equation (4) shows that the magnetic field $B_{\perp_{0}}$ and the size $r_{0}$ characterizing the accelerating region must satisfy $B_{\perp_{0}} r_{0} \leqslant 10^{12} n$.

Consider now some of the proposed mechanisms transferring angular momentum away from the neutron star in the light of this requirement. The basic model [3-6] consists of a rotating neutron star losing mass in a large magnetic field. The various theories differ on important questions such as the strength of the magnetic field and the amount of mass loss, etc.

In one class of model, the energy in the magnetic field is much greater than the energy of the mass flow away from the star, and the torque on the neutron star is produced by the radiation of very low frequency magnetic dipole radiation $[4,5]$. In this model the acceleration of charged particles occurs in a region having a size of the order of $10^{8} \mathrm{~cm}$, and a magnetic field strength $B_{0} \sim 10^{6}$ gauss, so that $B_{0} r_{0} \sim 10^{20}$. Hence, the electrons lose almost all their energy before escaping the region, regardless of the energies attained in the accelerating region. This model is a very efficient generator of synchrotron radiation but can not inject energy into the nebula in the form of relativistic electrons at anything like the required rate.

In the other type of model, the energy in the mass flow is much greater than the magnetic energy density, and the torque is produced by much the same process that 
slows down the sun and other stars. In this case, the flow of the plasma carries out the magnetic field lines to form a predominantly radial magnetic field structure which takes the form of Archimedean spirals as a result of the rotation of the neutron star. The radial extension of the magnetic field transfers angular momentum to the outflowing plasma and thereby results in a significant angular momentum loss. The energy density of the flow, which resides almost entirely in the protons, is fixed by the observed torque at about $10^{38} \mathrm{erg} / \mathrm{sec}$ ([6]; for more details, see [12]). This energy will be deposited in the nebula as a whole when the energy density of the flow drops to a value equal to the energy density of the ambient medium. For the Crab Nebula, the energy density of the ambient medium is of the order $10^{-8} \mathrm{erg} / \mathrm{cm}^{3}$ [13], so that the energy deposition must occur at a distance $r \sim 10^{17} \mathrm{~cm}$ from the neutron star. At this point, we might expect that a standing shock wave or some kind of transition region would exist. Fluctuations in the mass loss rate would result in fluctuations of the shock position and the generation of hydromagnetic waves.

Using this general picture, we can interpret the features which are observed in the active region near the center of the nebula. The most recent and exhaustive study of these features is given by Scargle [14]. He finds a permanent wisp which moves about but is confined to a definite region near the center of the nebula, about $10^{17} \mathrm{~cm}$ from the central star. It is proposed here that this wisp be identified with the transition region, or shock wave. In fact, Scargle states that the structure of the central wisp "is just the configuration which might be produced by a small object emitting ionized gas in all directions in an initially uniform magnetic field". A number of moving wisps that gradually damp out are also observed. These features may be interpreted as hydromagnetic waves generated by fluctuations in the mass loss rate.

Melrose [15] has shown that magnetic pumping in the moving wisps, considered to be hydromagnetic waves, can account for the large electron energies necessary to explain the observed X-ray emission. Note that $B_{0}^{2} r_{0} \sim 3 \times 10^{10}$, so that synchrotron losses are not a problem. In this way the extended source of $\mathrm{X}$-ray emission can be related to the observed rate of increase of the period of the pulsar. On the basis of this model there should be some correlation between a strong train of pulses and the appearance of a new wisp in the nebula, with an appropriate time lag.

The shock wave may also be a source of relativistic electrons. The rate at which relativistic particles are generated at the shock wave depends on the mass loss rate. For large mass loss rates $\left(>10^{17} \mathrm{gm} / \mathrm{sec}\right)$ the flow velocities are non-relativistic, whereas for the minimum mass loss consistent with Maxwell's equations $\left(\sim 2 \times 10^{7} \mathrm{gm} /\right.$ $\mathrm{sec}$ ) energies of the order of $10^{3} \mathrm{BeV}$ are attained [12]. If the electrons and protons share the energy equally in the shocked plasma, then $10^{3} \mathrm{BeV}$ electrons are produced. These electrons w ould have a lifetime of the order of 100 years and would emit optical radiation. Due to their long lifetime, they would diffuse throughout the nebula before emitting most of their energy. The observed optical luminosity of the nebula is uncertain because of the effects of interstellar absorption. Estimates range from $10^{36} \mathrm{erg} /$ sec to a value somewhat greater than $10^{38} \mathrm{erg} / \mathrm{sec}$ [16]. Of course, the electron energies might be considerably lower, and the radiation consequently shifted to lower 
frequencies. Observations in the far infrared should soon decide whether the Crab is anomalously bright there.

Concerning the X-ray emission from other pulsars, I have listed in Table I the rotational energy loss rate for the seven pulsars for which slowing down times have

TABLE I

Expected fluxes from pulsars

\begin{tabular}{lcll} 
Pulsar & $(\mathrm{d} E / \mathrm{d} t) \mathrm{n} . \mathrm{s}$. & Estimated distance & Flux at earth \\
\hline NP 0532 & $7 \times 10^{37} \mathrm{erg} / \mathrm{sec}$ & $2 \mathrm{kpc}$ & $2 \times 10^{-7} \mathrm{erg} / \mathrm{cm}^{2} \mathrm{sec}$ \\
PS 0833 & $8 \times 10^{35}$ & 0.468 & $3 \times 10^{-8}$ \\
CP 0950 & $1 \times 10^{32}$ & 0.048 & $4 \times 10^{-10}$ \\
CP 1133 & $1 \times 10^{31}$ & 0.026 & $2 \times 10^{-10}$ \\
CP 0834 & $2 \times 10^{31}$ & 0.253 & $3 \times 10^{-12}$ \\
CP 0808 & $<6 \times 10^{29}$ & 0.097 & $<6 \times 10^{-13}$ \\
CP 1919 & $3 \times 10^{30}$ & 0.093 & $3 \times 10^{-12}$
\end{tabular}

been measured. The computations assume that the moment of inertia is the same for all pulsars, $=10^{45} \mathrm{gm} \mathrm{cm}^{2}$. Using the slowing down rates and distances as given in [18] and [19], the total energy flux at earth is easily computed and is given in the fourth column of Table I. It would appear that only NP 0532 and PS 0833 are capable of producing detectable X-ray fluxes at earth. The recent observations of Gursky et al. [20] set a limit of $10^{-9} \mathrm{erg} / \mathrm{cm}^{2} \mathrm{sec}$ on the X-ray flux from Vela $X$ and rule out any identification of Vela XR-1 with PS 0833. However, the intensity of line emission from Vela $X$ is $\sim 3 \times 10^{-8} \mathrm{erg} / \mathrm{cm}^{2} \mathrm{sec}$ [21], so maybe all the energy goes into the excitation of line emission rather than the production of relativistic electrons.

Finally, consider briefly the case where the magnetic energy density is much greater than the particle energy density. It was shown above that if relativistic electrons were produced around such an object, they would immediately radiate all their energy by the synchrotron process. While such a model is not adequate to explain the X-ray source in the Crab Nebula, it is an attractive possibility for the compact X-ray sources such as Sco X-1.

A major difficulty for any model of Sco X-1 is the shape of the optical spectrum, which shows that the spectrum must turn over around $10^{15} \mathrm{~Hz}$. Requiring that this be due to synchrotron self-absorption fixes the angular diameter of the source in terms of the flux density $S_{v}\left(\mathrm{Wm}^{-2} \mathrm{~Hz}\right)$, and the magnetic field $B_{\perp}$ :

$$
\theta \cong 4 \times 10^{16} S_{v}^{1 / 2} B_{\perp}^{1 / 4} v^{-5 / 4} .
$$

Setting $v=10^{15} \mathrm{~Hz}$, and $S_{v}=10^{-26}[22]$, yields

$$
R \cong 2 \times 10^{6} B^{1 / 4} d_{\mathrm{kpc}} \mathrm{cm},
$$

where $d_{\mathbf{k p c}}$ is the distance to the source in kiloparsecs.

In order for the synchrotron process to produce the required luminosity we must 
have, for a uniform spherical source

$$
L_{\mathrm{syn}}=2 \times 10^{-15} B^{2} \gamma^{2} N \frac{4}{3} \pi R^{3}=L_{x} \simeq 10^{37} d_{\mathrm{kpc}}^{2} \mathrm{erg} / \mathrm{sec},
$$

where $N$ is the relativistic electron number density. It must satisfy the inequality

$$
N<B^{2} / 8 \pi \mathrm{mc}^{2} \gamma
$$

if the magnetic field energy is to be dominant. Equations (5)-(9) imply that we must have $R>4 \times 10^{7} d_{\mathrm{kpc}}^{16 / 17} \mathrm{~cm}$. On the other hand, if we require that the surface field on the neutron star $B_{\mathrm{ns}}$ be less than $10^{14}$ gauss, then $R<7 \times 10^{7} d_{\mathrm{kpc}} \mathrm{cm}$. Thus, $R$ must be about $10^{7} \mathrm{~cm}$. Choosing $d_{\mathrm{kpc}}=0.2$, we arrive at a model for Sco X-1 described by the following parameters:

$$
\begin{aligned}
& R \approx 10^{7} \mathrm{~cm} \\
& B=5 \times 10^{7} \text { gauss, } B_{\text {n.s }}=5 \times 10^{10} \text { gauss } \\
& \gamma=5 \times 10^{3} \quad N=6 \times 10^{5} \mathrm{~cm}^{-3}
\end{aligned}
$$

This discussion relates only to the emission mechanism and is independent of the assumed energy source. However, note that a rotating magnetic dipole [4,5] can produce $4 \times 10^{35} \mathrm{erg} / \mathrm{sec}$ if the frequency of rotation $\Omega=10^{3} \mathrm{rad} / \mathrm{sec}$, and the magnetic moment $M=5 \times 10^{28}$ gauss $\mathrm{cm}^{3}$. For this rotation frequency, the acceleration of electrons should occur around $R=c / \Omega \approx 3 \times 10^{7} \mathrm{~cm}$, in agreement with the above estimate for the size of the emitting region. Pulsed emission, if it occurs, should have a period of about $6 \mathrm{~m} \mathrm{sec}$.

\section{Acknowledgements}

This research has been supported by the National Aeronautics and Space Administration. It is a pleasure to acknowledge helpful discussions with A. J. Dessler and F. C. Michel.

\section{References}

[1] Haymes, R., Fishman, G., and Harnden, R.: 1969, Astrophys. J. (in press).

[2] Comella, J., Craft, jr., H. D., Lovelace, R. V. E., Sutton, J. E., and Tyler, G.: 1969, Nature 221, 453.

[3] Gold, T.: 1969, Nature 221, 25.

[4] Gunn, J. and Ostriker, J.: 1969, Nature 221, 454.

[5] Pacini, F.: 1968, Nature 219, 145.

[6] Michel, F. and Tucker, W.: 1969, Nature 223, 277.

[7] Finzi, A. and Wolf, R.: 1969, Astrophys. J. (Letters) 155, L107.

[8] Ginzburg, V. and Syrovatskii, S.: 1965, Ann. Rev. Astron. Astrophys. 3, 300 (Annual Rev., Palo Alto), and references cited therein.

[9] Haymes, R. C., Ellis, D. V., Fishman, G. J., Kurfess, J. D., and Tucker, W. H.: 1968, Astrophys. J. (Letters) 151, L9. - Peterson, L., Jacobson, A., Pelling, R., and Schwartz, D.: 1968, Canadian J. Phys. 47, 437. - Gorenstein, P., Kellogg, E., and Gursky, H.: 1969, Astrophys. J. 156, 315. Gould, R. J.: 1967, Amer. J. Phys. 35, 5.

[10] Burbidge, G. and Hoyle, F.: 1969, Nature 221, 847.

[11] Oda, M., Bradt, H., Garmire, G., Spada, G., Sreenkantan, B. V., Gursky, H., Giacconi, R., Gorenstein, P., and Waters, J. R.: 1967, Astrophys. J. (Letters) 148, L5. 
[12] Michel, F. C.: 1969, Astrophys. J. 158, 727.

[13] Woltjer, L.: 1958, Bull. Astron. Inst. Netherl. 14, 39.

[14] Scargle, J.: 1969, Astrophys. J. 156, 401.

[15] Melrose, D.: 1969, Astrophys. Space Sci. 4, 246.

[16] Geisel, S.: 1969, Rice University Preprint.

[17] Cameron, A. G. W.: 1969, 'Supernovae, Neutron Stars, and Pulsars'. Yeshiva University Preprint.

[18] Rohlfs, K., Grewing, M., and Mebold, U.: 1969, 'Improved Pulsar Distances'. Astron. Inst. Bonn Preprint.

[19] Grewing, M. and Priester, W.: 1969, 'Pulsar Periods as Age Indicators'. Astron. Inst. Bonn Preprint.

[20] Gursky, H., Kellogg, E., and Gorenstein, P.: 1968, Astrophys. J. (Letters) 154, L71.

[21] Milne, D.: 1968, Australian J. Phys. 21, 201.

[22] Neugebauer, G., Oke, J., Becklin, E., and Garmire, G.: 1969, Astrophys. J. 155, 1. 